\title{
Update of EUV Source Development Status for HVM Lithography
}

\author{
Hakaru Mizoguchi, Krzysztof M Nowak, Hiroaki Nakarai, Tamotsu Abe, Takeshi Ohta, Yasufumi Kawasuji, \\ Hiroshi Tanaka, Yukio Watanabe, Tsukasa Hori, Takeshi Kodama, Yutaka Shiraishi, Tatsuya Yanagida, \\ Tsuyoshi Yamada, Taku Yamazaki, Shinji Okazaki and Takashi Saitou
}

\author{
Gigaphoton Inc. Hiratsuka facility: 3-25-1 Shinomiya Hiratsuka Kanagawa, 254-8567,Japan \\ hakaru_mizoguchi@gigaphoton.com
}

\begin{abstract}
Carbon-dioxide laser driven, Sn-fueled, laser-produced-plasma (LPP) extreme UV (EUV) source emitting at $13.5 \mathrm{~nm}$ wavelength is currently regarded as a most promising route to EUV power levels required by high-volume-manufacturing (HVM) EUV lithography. We at Gigaphoton have been developing the $\mathrm{CO}_{2}$-Sn-LPP EUV light source since 2003. Along the way a range of unique, original technologies has been developed, such as a combination of ns-pulse $\mathrm{CO}_{2}$ laser and Sn droplets, dual-wavelength laser target preconditioning for an increased $\mathrm{CO}_{2}$-to-EUV conversion efficiency $(\mathrm{CE})$ and a plasma debris mitigation using a magnetic field, to name a few of them. The theoretical and experimental data accumulated so far have clearly showed the advantage of our proposed strategy. We report engineering data obtained from our prototype system \#2, such as $43 \mathrm{~W}$ average, clean EUV power at $100 \mathrm{kHz}$ pulse repetition rate. Also, high CE value of $3.9 \%$ was demonstrated at $20 \mathrm{kHz}$ repetition rate. Based on these achievements we are developing our first production source for HVM codenamed "GL200E". We project to reach EUV power level of 250W from currently available $\mathrm{CO}_{2}$ laser power of about $20 \mathrm{~kW}$. The development works aiming at higher $\mathrm{CO}_{2}$ laser (and EUV) power are currently in progress in cooperation with Mitsubishi Electric.

DOI: 10.2961/jlmn.2016.02.0021
\end{abstract}

Keywords: LPP, EUV, light source, lithography, semiconductor, $13.5 \mathrm{~nm}$, HVM

\section{Introduction}

Extreme ultraviolet (EUV) light source and wafer exposure tool technologies are being developed concurrently. In 2007') ASML has shipped its first " $\alpha$-demo tool" and Nikon shipped its own EUV-1 system in 20082), both equipped with an early $10 \mathrm{~W}$ EUV source. In the beginning of 2011 ASML has shipped the " $\beta$-tool", NXE-3100 employing 100W EUV light source. ${ }^{3) 4)}$

Presently, the " $\gamma$-tool" generation of EUV exposure system, suitable for the high-volume-manufacturing (HVM), is being developed. A requirement for the clean EUV power is set as $250 \mathrm{~W}$ at an intermediate focus (IF) plane of the exposure tool, "clean" meaning that the $\mathrm{CO}_{2}$ light and the other out-of-band radiation components are completely filtered out. Several machines of that kind have already been shipped in 2013, but their demonstrated EUV power levels did not exceed 50W.

Since 2003 we have been developing the carbon dioxide $\left(\mathrm{CO}_{2}\right)$ laser produced tin $(\mathrm{Sn})$ plasma $\left(\mathrm{CO}_{2}-\mathrm{Sn}-\mathrm{LPP}\right)$ EUV light source of $13.5 \mathrm{~nm}$ wavelength, which is considered as the most promising solution to produce high power $(>200 \mathrm{~W})$ light source required by HVM EUV lithography $($ EUVL $){ }^{5) 677)}$ We have chosen the $\mathrm{CO}_{2}$-Sn-LPP-EUV variant because of its relatively high efficiency, power scalability, and spatial freedom it provides around the plasma location. The theoretical ${ }^{8)}$ and experimental ${ }^{9)}$ data have clearly demonstrated that the combination of $\mathrm{CO}_{2}$ laser and $\mathrm{Sn}$ plasma can realize high conversion efficiency (CE) into $13.6 \mathrm{~nm}$ EUV light contained essentially within $2 \%$ bandwidth.

We have demonstrated in the past, using our $2 \mathrm{~Hz} \mathrm{R} \& \mathrm{D}$ EUV system, the advantage of $\mathrm{CO}_{2}$ laser as a plasma driver in terms of high CE values $>4.7 \%$. We have made efforts to incorporate these R\&D results into a HVM-worthy proto- type operating at $100 \mathrm{kHz}$ pulse repetition rate. Several technical challenges were faced, such as a sufficiently stable operation of a droplet generator, a stability of the $\mathrm{CO}_{2}$ laser beam ${ }^{10)}$ at high average power, and a sufficiently accurate control of target irradiation conditions. In the present paper we provide an update of our latest progress in these areas.

2. LPP EUV light source system 2.1 System concept

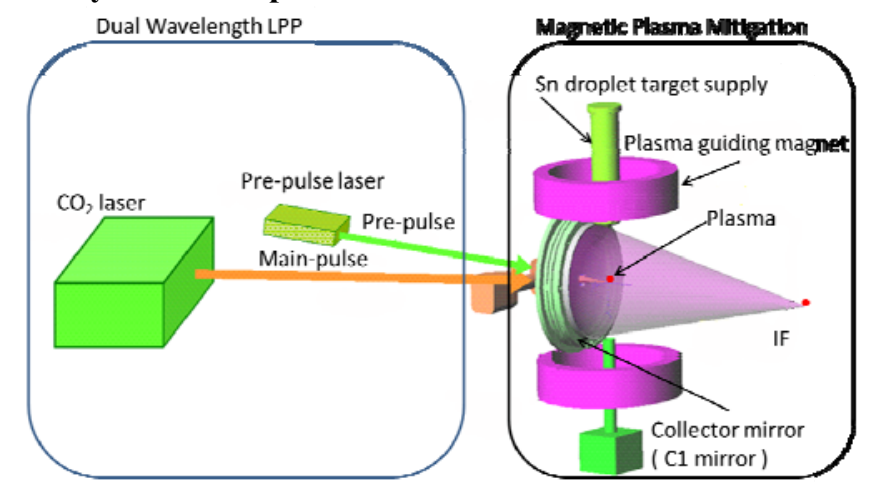

Fig. 1 Concept of Gigaphoton's HVM EUV light source.

The concept of Gigaphoton's HVM EUV light source is shown schematically in Fig.1. The concept is characterized by 5 key, patent-pending technologies:

(1) High ionization rate and high $\mathrm{CE}$ achieved by a combined $\mathrm{CO}_{2}$ and solid-state laser irradiation of the Sn target.

(2) Original, hybrid $\mathrm{CO}_{2}$ laser system featuring a high repetition rate, solid-state seeded, short 20ns pulse oscillator, amplified by a system of commercial, multi-kW cwamplifiers. 
(3) Accurate control of target irradiation conditions.

(4) Mitigation of Sn debris with a super-conductive magnet.

(5) Highly-efficient filtration of out-of-band radiation using a grating structured EUV collector mirror.

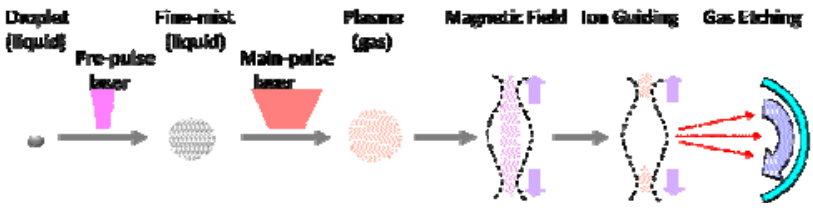

Fig. 2 Concept of EUV emission \& Sn mitigation processes

The concept of Sn debris mitigation with a magnetic field is shown in Fig. 2. At first, the tin droplet is irradiated with the pre-pulse laser and is rapidly converted from a high-density liquid into a lower-density mist of sub-micron sized, liquid particles. Next, after a certain time delay, the mist cloud is irradiated by a pulsed $\mathrm{CO}_{2}$ laser beam and turned into a high-temperature plasma, where the $\mathrm{Sn}$ ions undergo a multiple-order ionization and the plasma emits (among other wavelengths) $13.5 \mathrm{~nm}$ EUV light during recombination processes. The residues of the plasma, which remain after the EUV emission is finished, are eventually scattered inside the vessel if no precautions are taken. In order to prevent the collector mirror from being contaminated, Sn plasma (and its products) has to be constrained spatially. Thanks to a high degree of ionization, most of the Sn ions can be trapped by the magnetic field in the trajectories of so-called Larmor movements. To enhance EUV energy and to maximize Sn debris mitigation, the number of Sn ions should be maximized in these laser heating processes.

\subsection{Pre-pulse technology ${ }^{11) 12) 13)}$}

\subsubsection{Case of ns pre-pulse}

We have found that the initial droplet becomes highly fragmented after the pre-pulse irradiation. By volume, the fragments constitute a major part of the preconditioned $\mathrm{Sn}$ target. The diameter of the fragments can reach as much as a few micrometers, as evidenced by a stroboscopic, shadowgraph metrology utilizing a few-nanosecond, pulsed back illuminator and a CCD camera equipped with a highresolution telescope.

Fig. 3 shows the shadowgraphs of the fragments after the pre-pulse laser irradiation of the $20 \mu \mathrm{m}$ droplet, which was irradiated by the pre-pulse laser arriving from the left hand side in the image. After the laser irradiation, the cloud of fragments expanded in diameter while travelling in a direction being a vector sum of initial droplet momentum and a net momentum increase resulting from the laser irradiation. Fig. 3(a) shows a case where (we believe) the vaporization of $\mathrm{Sn}$ fragments upon $\mathrm{CO}_{2}$ laser irradiation was seen to be nearly complete. In contrast, Fig. 3(b) below shows shadowgraphs obtained in a situation of non-optimal cloud condition, which resulted in easily observable fragment residues.

The experiments have shown that some optimal conditions exist for a near-perfect vaporization and ionization of Sn fragments and that they can be obtained by an appropriate selection of irradiation conditions, such as pre-

pulse/main-pulse combination and a sufficiently small Without main-pulse laser

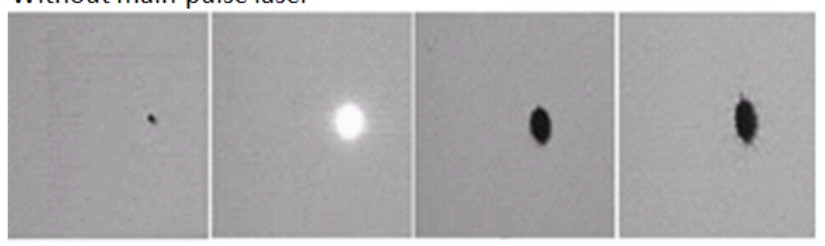

With main-pulse laser

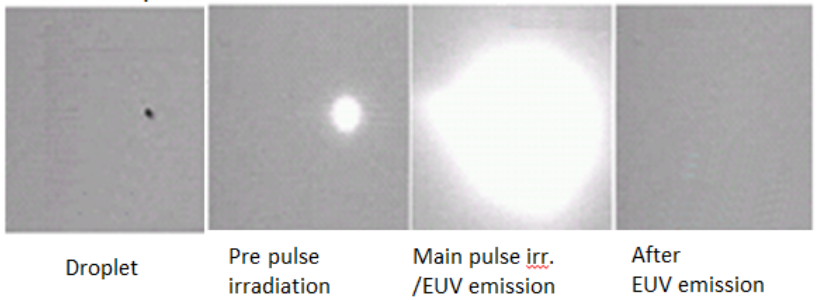

(a)

Without main-pulse laser

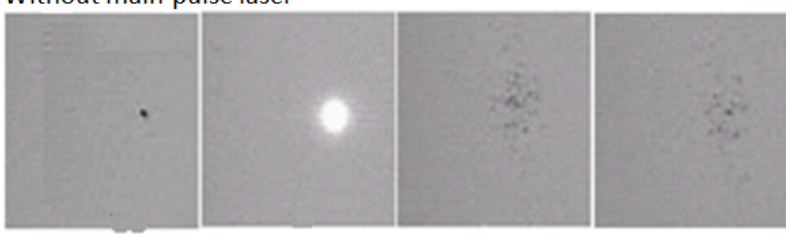

With main-pulse laser

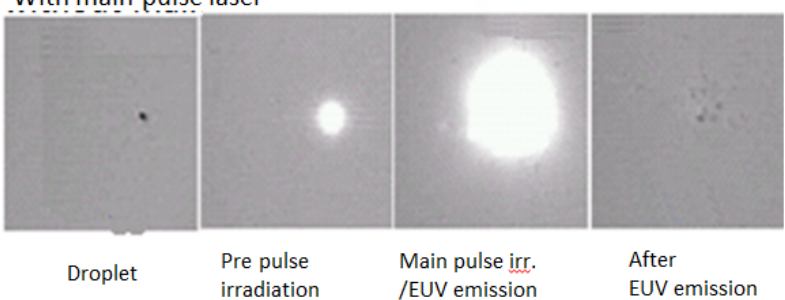

(b)

Fig.3. Shadowgraphs of optimal, double pulse plasma fragments, (a); and non-optimal fragments, (b).

droplet diameter. We have therefore investigated the conversion efficiency (CE) as a function of the droplet diameter and the pre-pulse laser irradiation. Fig. 4 shows the results indicating that high $\mathrm{CE}$ can be obtained even with a droplet size below $20 \mu \mathrm{m}$. The pre-pulse laser irradiation was seen to be a key requirement for obtaining higher CE. The CE reached $3.3 \%$ with the $20 \mu \mathrm{m}$ in diameter droplet following the optimization of the pre-pulse laser conditions. We have thus found a way to reduce the debris without the degradation of CE by using droplets of diameter $<20 \mu \mathrm{m}$ and a proper combination of target irradiation conditions.

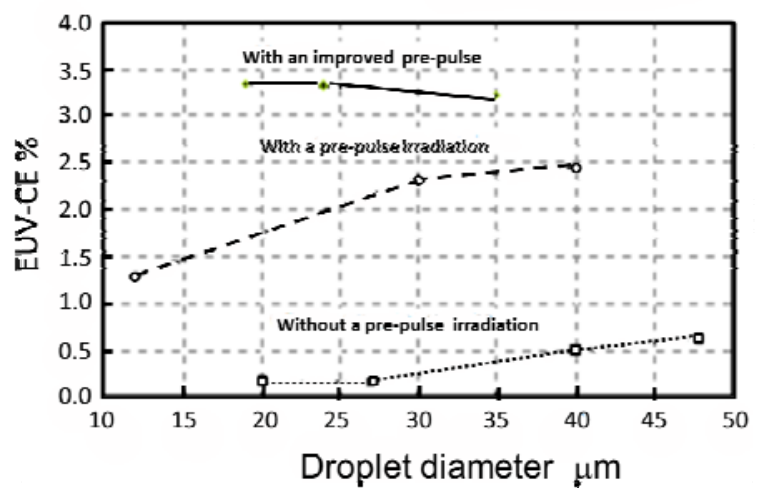


Fig. 4. Conversion efficiency vs. droplet diameter.

\subsubsection{Case of ps pre-pulse}

High CE of $4.7 \%$ has been demonstrated in our R\&D setup, as shown in Fig.5 (red upper line), following an implementation of ps-pulse target preconditioning of $20 \mu \mathrm{m}$ droplets. These basic studies, supporting the accompanying theoretical considerations, have contributed to the development of the high-power production system and to the basic design for further EUV power scaling.

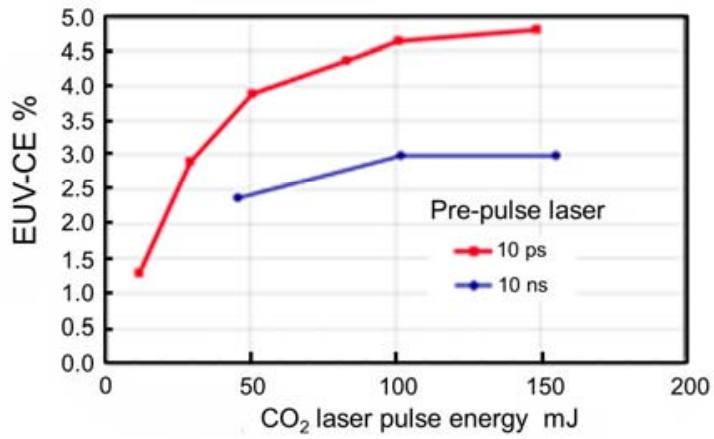

Fig. 5. Higher CE was obtained with ps pre-pulse.

The significant increase of $\mathrm{CE}$, following a picosecond pre-pulse, is explainable by a difference of mist formation and expansion mechanisms associated with the nanosecond and picosecond timescales. This high CE technology enables $250 \mathrm{~W}$ EUV source from a moderate $20 \mathrm{~kW} \mathrm{CO}$ laser power.

The relative proportion of ions and neutrals, or the ionization ratio, is an important parameter in the process of low-debris, high-CE EUV emission. A spectroscopic, laserinduced-fluorescence (LIF) method was employed to directly measure the ionization ratio. The LIF imaging of the Sn atoms has several advantages, such as a selectivity of species and high sensitivity. A schematic view of the experimental setup is shown in Fig. 6. The Sn atoms were excited by a third harmonics, narrow-band Ti:sapphire laser tuned to the $5 \mathrm{p}^{2}{ }^{3} \mathrm{P}_{0}-6 \mathrm{~s}^{3} \mathrm{P}^{\mathrm{o}}{ }_{1}(286.3 \mathrm{~nm})$ transition line of atomic tin. The fluorescence from the $5 \mathrm{p}^{2}{ }^{3} \mathrm{P}_{2}-6 \mathrm{~s}{ }^{3} \mathrm{P}^{\mathrm{o}}{ }_{1}$ $(317.5 \mathrm{~nm})$ transition was observed through a band-pass filter with an image-intensified CCD (ICCD) camera. Twodimensional atom distributions were obtained using a thin, sheet-like laser beam that probed a plane parallel to the direction of laser irradiation (plane normal was perpendicular to the axis of the laser beam).

The amount and the distribution of Sn neutral atoms after the pre-pulse laser irradiation in a magnetic field of certain strength were observed experimentally. Fig. 7 shows one example result of the LIF measurements. After an irradiation by a $\mathrm{CO}_{2}$ laser of insufficient energy, the neutral atoms and the fragments manifested themselves by a strong fluorescence (blue light on image). In contrast, no neutral atoms and fragments were observed after $\mathrm{CO}_{2}$ laser irradiation with high enough energy.

The ionization ratio can be calculated by spatial integration of the fluorescence signals. The calculated ionization ratio vs. $\mathrm{CO}_{2}$ laser energy is shown in Fig.8 and $\mathrm{CE}$ vs. $\mathrm{CO}_{2}$ laser energy is shown in Fig.9, having the pre-pulse duration as a parameter in both.

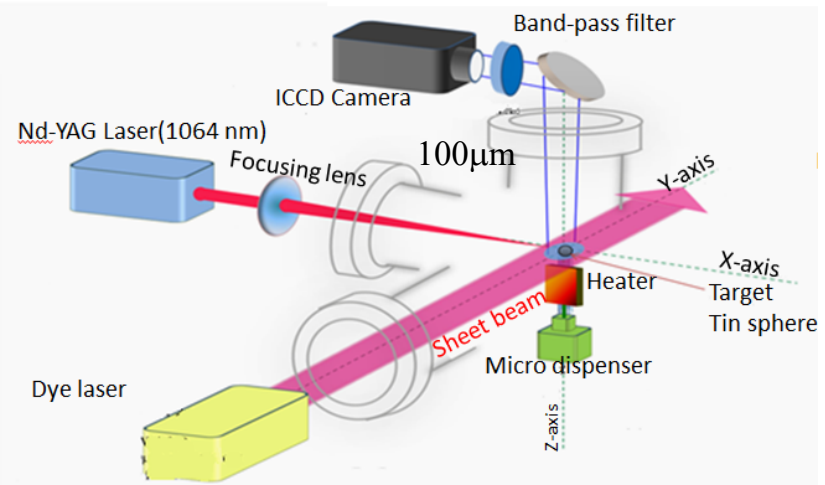

Fig.6 Ionization ratio measurement.

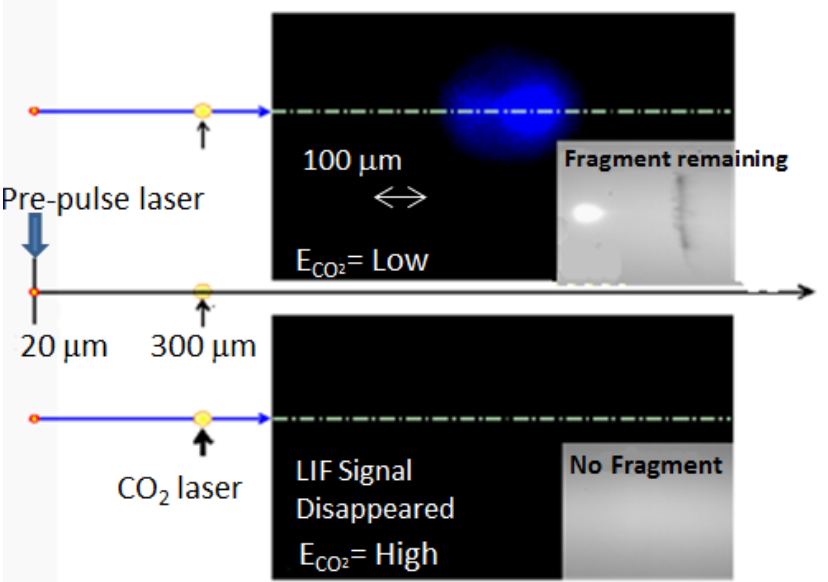

Fig. 7 Fluorescence signal from neutral $\mathrm{Sn}$ atom.

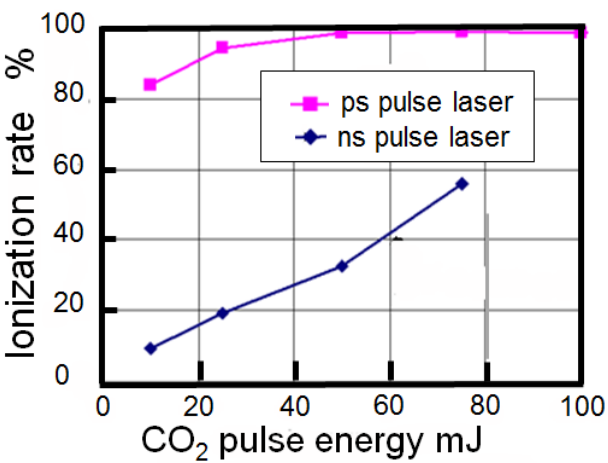

Fig. 8 Ionization ratio vs. $\mathrm{CO}_{2}$ laser energy.

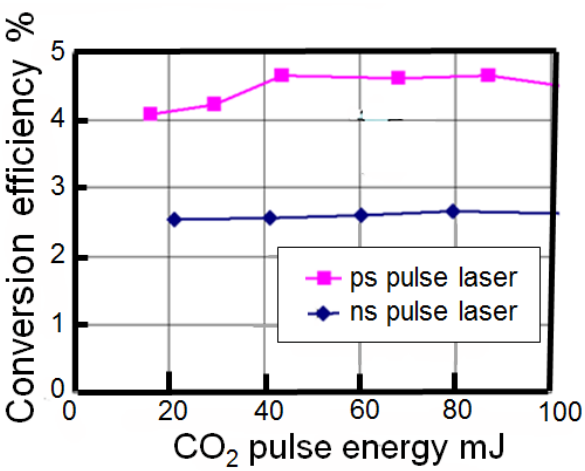

Fig. 9 Conversion efficiency vs. $\mathrm{CO}_{2}$ laser energy with a pre-pulse duration as a parameter.

The ionization ratio obtained with the ns pre-pulse is 
shown in Fig.8. This data indicates that a high ionization ratio can be obtained when $\mathrm{CO}_{2}$ laser energy is in excess of $100-200 \mathrm{~mJ}$. In contrast, the ionization ratio was near-unity even with the $\mathrm{CO}_{2}$ pulse energy below $20 \mathrm{~mJ}$ in the case of 10 ps pre-pulse. It is good news, because a lower power, $20 \mathrm{~kW} \mathrm{CO} \mathrm{CO}_{2}$ laser seems sufficient to achieve the planned 250W EUV power level. The debris mitigation system may also work better in the situation of lower power operation.

\subsection{Droplet generator \& magnetic-mitigation technology}

The tin injected into the active region has to be almost fully captured in order to mitigate the detrimental effects the deposition and erosion by energetic ions have on the collector mirror lifetime. Our idea of magnetic-field-assisted technique of Sn debris mitigation is simple. A strong magnetic field traps the $\mathrm{Sn}$ ions and electrons that remain after the process of EUV emission from Sn plasma. The maximization of the ionization ratio is therefore essentially important not only for the conversion efficiency, but also for an effective management of Sn debris. In a perfect case of all the Sn atoms becoming ionized, all debris could possibly be guided along the magnetic flux. Also, some neutral atoms could be trapped and guided following a charge exchange with the ions. ${ }^{11)}$

In reality, however, not all the Sn atoms and ions can be effectively trapped in the magnetic field. In order to accommodate for that real-life problem, our system is also equipped with a chemical etching mechanism. With this mechanism the "rogue" Sn atoms and clusters, which have managed to deposit on EUV collector optics, viewports, chamber walls and other internal equipment, can be removed (Fig.10). 12)

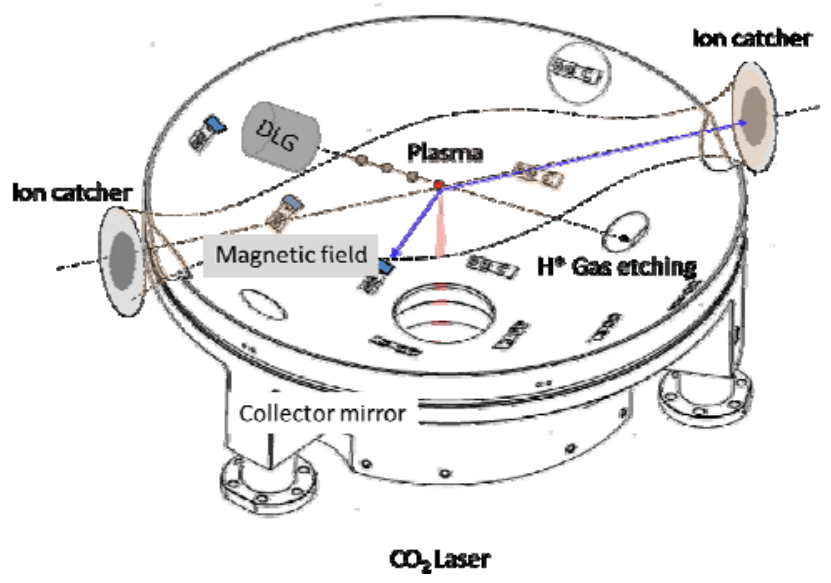

Fig. 10 Schematic view of the collector mirror and the mitigation system.

Numerous optimization studies have been carried out to determine the optimum performance of debris mitigation technique to match the operational conditions of a production EUV source. The beneficial combination of $20 \mu \mathrm{m}$ droplets and $10 \mathrm{ps}$ pre-pulse irradiation, leading to a near$100 \%$ ionization ratio, has been implemented in our $100 \mathrm{kHz}$ prototype system.

\subsection{1 $20 \mu \mathrm{m}$ diameter droplet generator}

A generation of a sufficiently small $\mathrm{Sn}$ droplet is particularly important in the process of Sn debris mitigation, because the amount of $\mathrm{Sn}$ should be minimized to what is absolutely necessary to obtain enough EUV photons without compromising the overall performance of a droplet generator. Recently we have succeeded to realize a stable generation of $20 \mu \mathrm{m}$ diameter droplets. The performance of our $20 \mu \mathrm{m}$ droplet generator is shown in Fig. 11 .
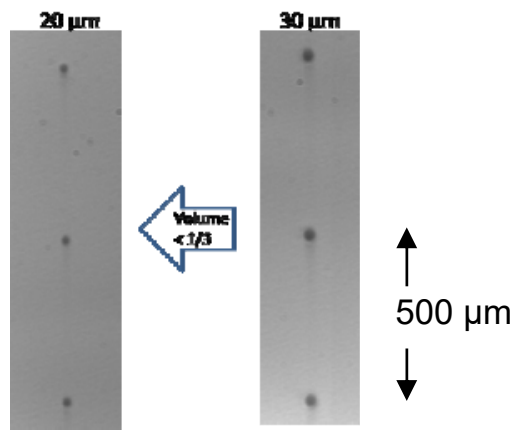

(a)

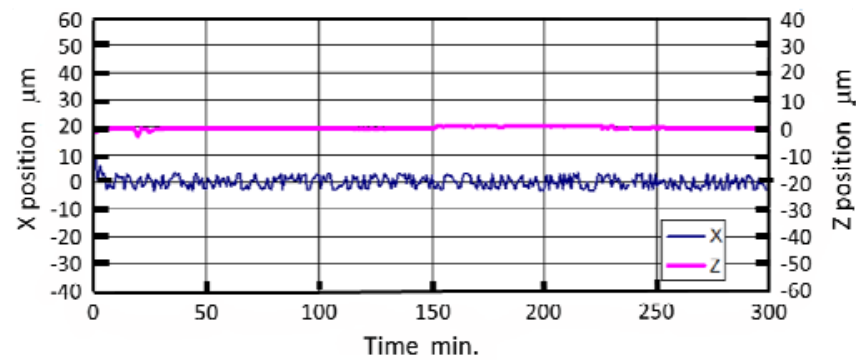

(b)

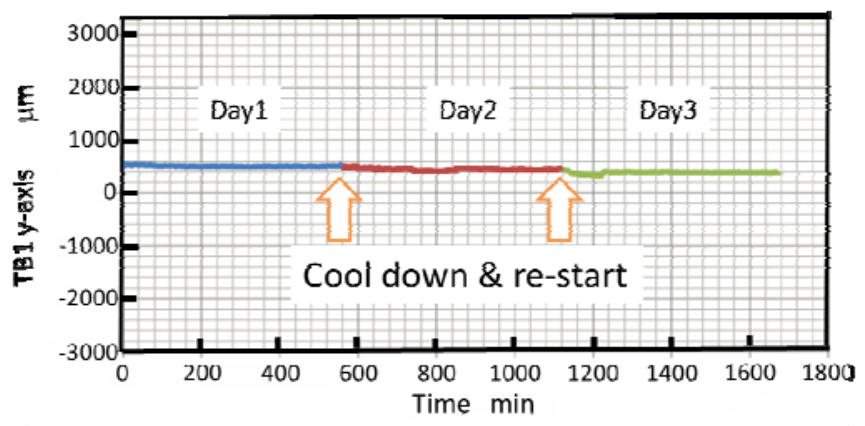

(c)

Fig. 11. Reduction of Sn mass by $3.3 \mathrm{x}$ achieved by a transition from $30 \mu \mathrm{m}$ to $20 \mu \mathrm{m}$ droplets, (a); Mid-term position stability, (b); and long-term position stability of droplet at laser irradition position, (c)

The liquid Sn droplets are generated by the generator nozzle that draws a liquid tin from a supply tank heated above the melting temperature of $\mathrm{Sn}(>231.93$ degree Celsius). The position of the droplet arriving at the laser irradiation spot is measured by a position sensor and the results are fed back to the droplet generator. This is very important commercially because the droplet position stability determines the overall position and energy stability of the EUV light source. The pre-pulse laser and $\mathrm{CO}_{2}$ laser irradiation positions are monitored also for the same reason by a set of dedicated sensors. 


\subsubsection{Debris dependency on droplet size}

A deposition of Sn layer only $1 \mathrm{~nm}$ thick (a few atomic layers) degrades the mirror reflectivity by $10 \%$, which has to be taken into consideration in the mirror lifetime specification. ${ }^{12)}$ A study aiming to determine a relationship between an amount and a character of the laser-produced debris and the droplet diameter was carried out. Several droplet diameters were used and a deposition rate in several locations on a dummy collector mirror was measured, as shown in Fig.12. Table 1 shows the results obtained at the A1 position. The deposition rate in case of the $20 \mu \mathrm{m}$ droplet, which contained nearly half the mass as compared to the $25 \mu \mathrm{m}$ droplet, was found to be five times smaller and around $0.1 \mathrm{~nm}$ per million pulses. During these experiments, however, an accuracy of laser targeting system was still an issue and, as a consequence, a fragment-type debris was observed and a standard deviation of recorded EUV energy was large, especially in the case of the smaller $20 \mu \mathrm{m}$ droplet. A problem of an insufficient control of the irradiation condition still remains to be solved.

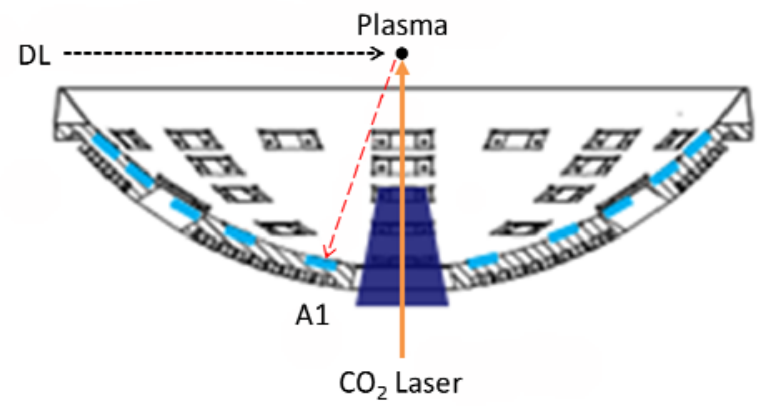

Fig.12 Dummy collector mirror was used to measure the spatial distribution of $\mathrm{Sn}$ deposition rates.

Table 1. Comparison of recorded deposition rates.

\begin{tabular}{|c|c|c|}
\hline & 25 um Droplet & 20 fm Draplet \\
\hline Pulse number & $152 \times 10^{6} \mathrm{pls}$ & $80 \times 10^{6} \mathrm{pls}$ \\
\hline $\mathrm{H}_{2}$ pressure & $15 \mathrm{~Pa}$ & $15 \mathrm{~Pa}$ \\
\hline EUV Energy $(3 \sigma)$ & $23.3 \%$ & $73.4 \%$ \\
\hline $\begin{array}{l}\text { A1 sample Center of collector } \\
\text { mirror (SEM } \times 10,000)\end{array}$ & & \\
\hline Sn deposition rate & $0.5 \mathrm{~nm} / \times 10^{6} \mathrm{pls}$ & $0.1 \mathrm{~nm} / \times 10^{6} \mathrm{pls}$ \\
\hline
\end{tabular}

\subsection{IR reduction technology on collector mirror ${ }^{17)}$}

The EUV light emitted by the plasma is collected by a multilayer mirror. Beside the EUV, the plasma emits also the accompanying thermal radiation and also reflects a significant fraction of the $\mathrm{CO}_{2}$ laser light. These undesired, out-of-band components are strongly reflected by the collector mirror, as shown in Fig.13.

In the past, several kinds of transmission filters were used to cut the out-of-band light. However, the performance of a transmission filter was limited by an optical absorption of the filter material itself, giving rise to heat management problems. We have devised a new type of filter, which has a diffractive, grating-like structure fabri- cated on the surface of the multilayer coating (patent pending). Fig. 14 shows schematically the principle of operation of this new filter. The IR light reflected from the multilayer coating creates an interference pattern at a focal plane such that the IR light can be absorbed by an aperture stop while the shorter wavelengths can pass with a low loss. A drawback of this kind of solution is a reduction of reflectivity of the collector mirror in the EUV band. Fig. 15 shows the results of EUV reflectivity measurements of a collector mirror featuring the IR-rejection filter. The data show that the reduction of EUV reflectivity was not critical and only around $4 \%$ throughout the whole of the collector mirror surface.

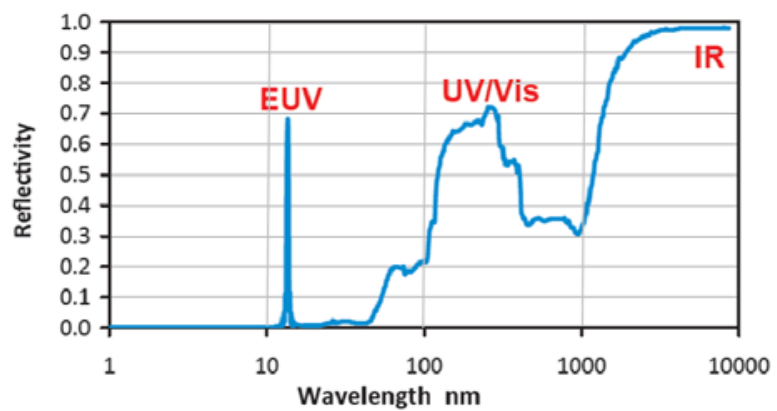

Fig. 13. Typical reflectivity of the multi-layer EUV collector mirror.

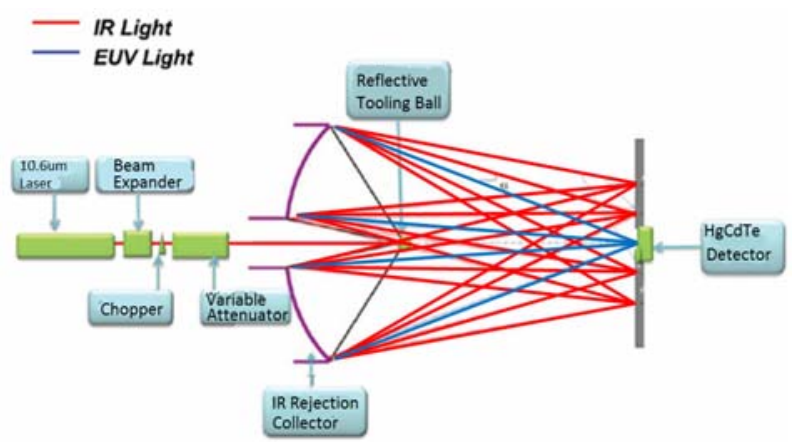

Fig. 14. Schematic of a test setup used to evaluate the performance of new type, diffractive IR filter.

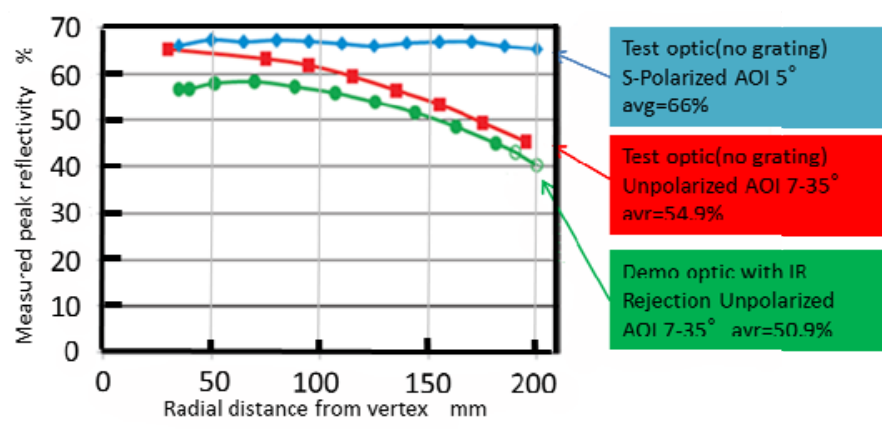

Fig. 15. Measurement result of EUV reflectivity as a function of radial position on the collector mirror featuring the IR rejection filter (green line with circles).

Fig. 16 presents an experimental confirmation of the filter operation, obtained in the test setup measurement depicted in Fig.14. A significant reduction of the IR light by more than $99 \%$ was recorded, which was an excellent result ${ }^{17)}$. 


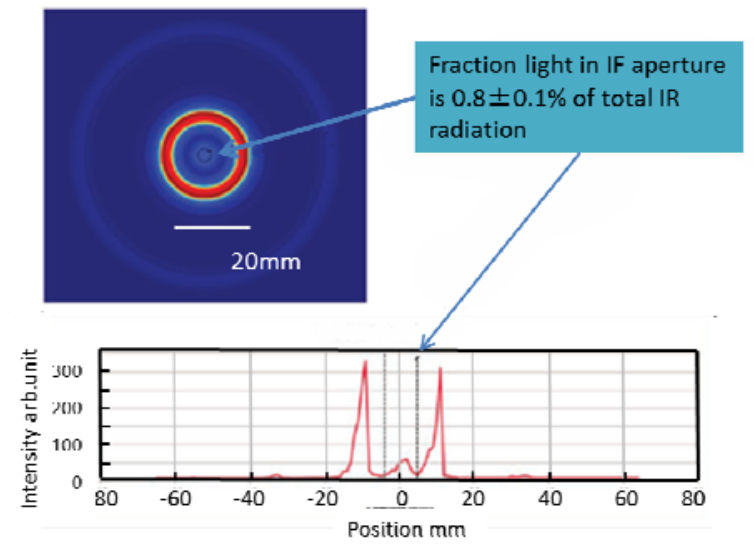

Fig. 16 Simulated (top) and measured (bottom) diffraction pattern created at the focal plane of the IR-rejecting collector mirror ${ }^{17)}$

\subsection{EUV light source system}

The first generation of our projected HVM EUV light source comprises of $100 \mathrm{kHz}$ rep-rate, $20 \mathrm{~kW} \mathrm{CO}_{2}$ laser, the $20 \mu \mathrm{m}$ droplet generator and the magnetic field debris mitigation. An overview of the light source is shown in Fig 17.

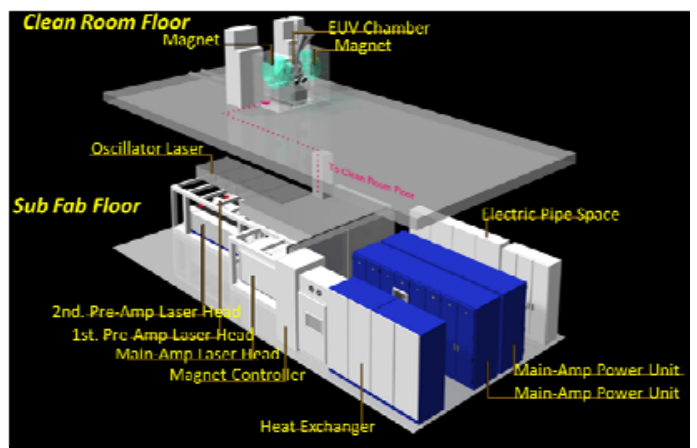

Fig. 17250 W LPP EUV Light Source System.

The EUV chamber vessel contains the droplet generator, the collector mirror, and several vacuum pumps. The main function of the EUV chamber vessel is to maintain high level vacuum environment around the EUV-emitting plasma and to facilitate and implement the extraction of plasma remnants and Sn debris in order to maximize the performance and lifetime of the EUV collector mirror. In order to implement the $\mathrm{Sn}$ debris mitigation, a pair of superconducting, magnetic coils is arranged on two opposite sides of the vessel.

The EUV chamber should be located closely next to a scanner in order to minimize the loss of EUV power. The clean room floor of the EUV exposure tool is mainly occupied by the scanner, therefore the $\mathrm{CO}_{2}$ laser system is located on a different floor than the scanner (usually below) to minimize the overall footprint in the clean room area.

\section{System Test and Result 3.1 Prototype systems}

We have constructed two prototype EUV source systems so far, called "proto \#1" and "proto \#2". The configuration and specification data of both systems are shown in
Table 2, along with the data describing the production system under development ("customer beta"). A major difference between the three systems is the $\mathrm{CO}_{2}$ laser power and the output angle of EUV beam line, while other specifications are essentially the same.

A construction of "proto \#1" system was commenced in 2011 ${ }^{13)}$. A suitable system technology had been developed since then and an extensive testing of the building blocks was undertaken. We have achieved 34W clean EUV power (@ I/F) using the "proto \#1" system. There were two major issues however, one was a necessity to improve the long-term stability of the droplet generator, and the other was a need for more $\mathrm{CO}_{2}$ laser power.

Table 2. Characteristic data of EUV LPP source systems under development .

\begin{tabular}{|c|c|c|c|c|}
\hline \multicolumn{2}{|c|}{ Operational Specification (target) } & Proto \#1 & Proto \#2 & Custnmar Reta Unlt \\
\hline \multirow{5}{*}{ Performance } & Luv Power & $25 \mathrm{w}$ & ssow & $250 \mathrm{w}$ \\
\hline & CE & $3 \%$ & $1 \%$ & $1 \%$ \\
\hline & Pulse rate & $100 \mathrm{kHz}$ & $100 \mathrm{kHz}$ & $100 \mathrm{kHz}$ \\
\hline & Output angle & horizontal & $\begin{array}{c}62 \text { degrees } \\
\text { upper } \\
\text { (matched to } \\
\text { NXE) }\end{array}$ & $\begin{array}{l}62 \text { degrees upper } \\
\text { (matched to NXF) }\end{array}$ \\
\hline & Avallahulity & $\begin{array}{c}\text { 1week } \\
\text { (opcration timc) }\end{array}$ & $\begin{array}{c}1 \text { wectik } \\
\text { (nperation } \\
\text { time) }\end{array}$ & $>75 \%$ \\
\hline \multirow{3}{*}{ Texturnolopy } & $\begin{array}{l}\text { Droplct } \\
\text { Generator }\end{array}$ & $20-25 \mu \mathrm{m}$ & $20 \mu \mathrm{m}$ & $<20 \mu \mathrm{m}$ \\
\hline & $\begin{array}{c}\text { CO2 Laser } \\
\text { Pre-pulse Laser }\end{array}$ & $\begin{array}{c}>8 \mathrm{~kW} \\
\text { plcosecond }\end{array}$ & $\begin{array}{c}>12 \mathrm{~kW} \\
\text { plcosecond }\end{array}$ & $\begin{array}{c}25 \mathrm{~kW} \\
\text { PIcosecond }\end{array}$ \\
\hline & Debris Mitigation & $\begin{array}{l}\text { Validation of magnetic } \\
\text { mitieation in systcm }\end{array}$ & 10days & 15days \\
\hline
\end{tabular}

\subsection{Latest experimental result of Proto \#2 system}

The knowledge gained during the development period of "proto \#1" system enabled us to undertake a construction of an upgraded "proto \#2" system. It was initiated in a second half of 2013 and the system became operational in the beginning of 2014. Substantial progress has been made since then. The stability of the droplet generator was improved dramatically and the $\mathrm{CO}_{2}$ laser power at target was raised from $5 \mathrm{~kW}$ to $8 \mathrm{~kW}$

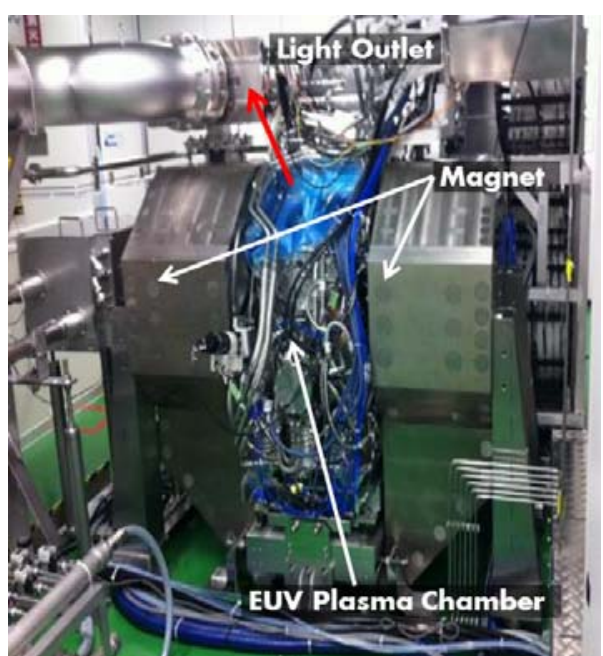

Fig. 18 EUV chamber of proto \#2 system

A picture of "proto \#2" EUV chamber is shown in Fig. 18. The enclosures housing the pair of superconducting magnets are visible. The chamber design was made such 
as to assure the mechanical compatibility with the EUV exposure tool while at the same time to provide an easy access to all components for the maintenance purposes.

The first experimental result of Proto \#2 is shown in Fig.19 (a) and (b). 16.9W of clean EUV was recorded at the intermediate focus point (IF) in burst mode of operation characterized by $20 \mathrm{kHz}$ repetition rate of EUV pulses and $50 \%$ burst duty cycle $(\mathrm{ON} / \mathrm{OFF}=0.5 \mathrm{sec} / 0.5 \mathrm{sec})$. CE was measured to be $3.9 \%$. Also, $43.4 \mathrm{~W}$ clean EUV power was recorded at $100 \mathrm{kHz}$ repetition rate of EUV pulses and $50 \%$ burst duty cycle $(\mathrm{ON} / \mathrm{OFF}=0.1 \mathrm{sec} / 0.1 \mathrm{sec})$. In this case the CE of $2.4 \%$ was obtained. The substantial difference of CE values between these two modes of operation is under investigation at present.

(a)

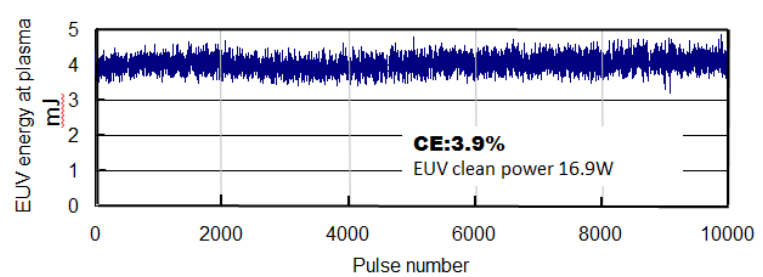

(b)

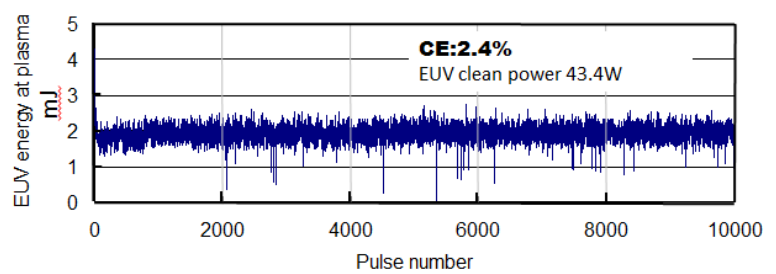

Fig.19 EUV emission data at (a) conversion efficiency of $3.9 \%$ and EUV clean power of $16.9 \mathrm{~W}$, and (b) conversion efficiency of $2.4 \%$ and EUV clean power of $43.4 \mathrm{~W}$.

\subsection{Scale-up plan for $\mathrm{CO}_{2}$ laser power}

The achievement of higher $\mathrm{CE}$ values must be accompanied by further improvement of available $\mathrm{CO}_{2}$ laser drive power in order to reach our EUV power target of $250 \mathrm{~W}$. For that purpose a $20 \mathrm{~kW}$ master-oscillator-power-amplifier (MOPA) laser system is required.

Fig.20 shows current status and a future of our $\mathrm{CO}_{2}$ laser driver development. "Proto \#1" system has delivered stable $5 \mathrm{~kW}$ and "proto \#2" system $8 \mathrm{~kW}$ at present. Both systems are very similar except for one more power amplifier in the "proto \#2" system. New MOPA system is designed to produce $20 \mathrm{~kW}$ average power level.

The MOPA design consists of a hybrid $\mathrm{CO}_{2}$ laser master oscillator (OSC) and a system of RF-discharge-excited, $\mathrm{CO}_{2}$ laser amplifiers. The amplifier system in turn consists of low-power, high-gain post-amplifiers (Osc-AMP), highgain multi-kW power preamplifier (Pre-AMP) and a cascade of power amplifiers (main-AMP). The commercial $\mathrm{cw}-\mathrm{CO}_{2}$ lasers were used as the amplifiers after some minor modifications. The RF-excitation is a commonly employed scheme in axial flow, transverse flow or diffusion-cooled, slab-waveguide configurations. It allows for a high repetition rate, pulsed operation demanded by the EUV power requirement.

The master oscillator (OSC) is an original, solid-state seeded, multi-pass amplifier custom-built around a small, slab-waveguide, RF-excited $\mathrm{CO}_{2}$ laser ${ }^{19) 20}$. It is character- ized by a multi-line operation intended for an enhanced energy extraction in the power amplifiers. The seed pulses are generated independently by a set of four solid-state, quantum-cascade lasers ${ }^{21}$ (QCL) allowing for an asynchronous, on-demand generation of highly stable pulses of a width adjustable electronically from about 12 to $40 \mathrm{~ns}$, and with repetition rates from practically 0 to $100 \mathrm{kHz}^{18)}$.

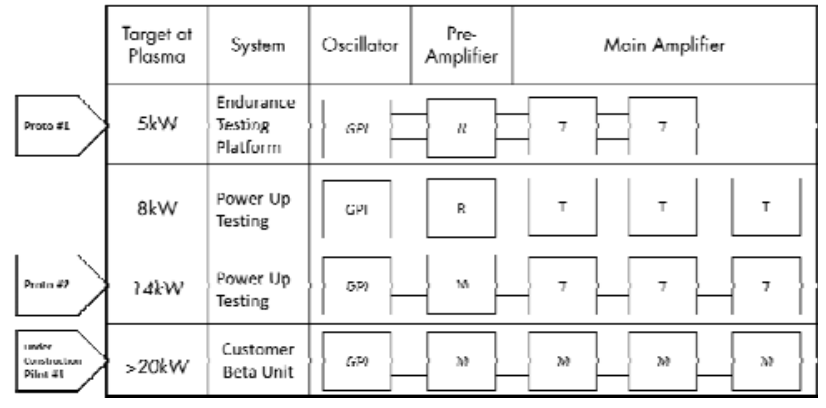

Fig. 20. Scale-up plan for the driver $\mathrm{CO}_{2}$ laser power.

The output from the master oscillator is pre-amplified to about $150 \mathrm{~W}$ by a system of custom-design, multi-pass, small slab-waveguide, amplifiers (Osc-AMP). Subsequently, the pre-amplifier (Pre-AMP), which is custom-built on a largest available slab-waveguide laser ${ }^{22}$, boosts the power to a level of $2 \mathrm{~kW}(20 \mathrm{~mJ}$ at $100 \mathrm{kHz})$. The following two or three main-amplifiers (main-AMP) amplify the pulse emerging from the PreAMP to a final $5 \mathrm{~kW}$ and $8 \mathrm{~kW}$ at the target location, in "proto \#1" and "proto \#2" systems respectively. The MOPA laser system is operable from low duty mode $(2 \%)$ to full duty mode $(100 \%)$.

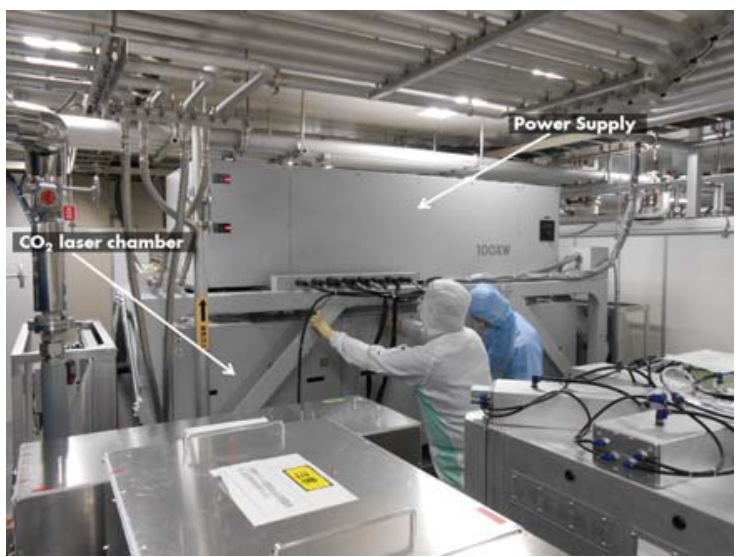

Fig. 21. Installation of new pre-amplifier in the "proto \#2" $\mathrm{CO}_{2}$ laser system.

Since 2011 we have had a support of New Energy and Industrial Technology Development Organization (NEDO) to aid our development of a new $\mathrm{CO}_{2}$ amplifier in cooperation with Mitsubishi Electric ${ }^{14) 16)}$. As a result of this successful effort, new Pre-AMP and main-AMP designs emerged, enabling us to make a further improvement of the $\mathrm{CO}_{2}$ laser power. "Proto $\# 2$ " is currently undergoing an upgrade of the Pre-AMP to a new type ${ }^{23)}$ capable of $4-5 \mathrm{~kW}$ output $(40-50 \mathrm{~mJ}$ at $100 \mathrm{kHz})$ leading to a projected $14 \mathrm{~kW}$ power at plasma point. Fig.21 shows a picture of this new pre-amplifier taken during installation activities in our laboratory. 
A development work on a new power amplifier system aiming at the $20 \mathrm{~kW} \mathrm{CO}$ power level at plasma is ongoing in co-operation with Mitsubishi. In 2013 we succeeded to demonstrate $21 \mathrm{~kW}$ output power with an engineering prototype (equivalent to "Pilot \#1" in Fig.20) consisting entirely of Mitsubishi-developed, fast-transverse-flow, RFdischarge excited laser units ${ }^{15) 16(18)}$.

\section{Conclusions}

We have reported on a progress of component technology of EUV light source system. $20 \mu \mathrm{m}$ droplets at $100 \mathrm{kHz}$ emission rate were successfully generated and a dramatic reduction of debris deposition rate on the collector mirror was demonstrated. An IR rejection technology was incorporated into the collector mirror by the maker and shown to be capable of very effective $>99 \%$ reduction of undesired IR radiation. We have demonstrated a successful generation of EUV in our "proto \#2" system and also 200W EUV power at plasma and $43 \mathrm{~W}$ clean EUV power at the intermediate focus plane at $100 \mathrm{kHz}$ pulse repetition frequency. High conversion efficiency (CE) of $3.9 \%$ (at $20 \mathrm{kHz}$ ) was confirmed using the pico-second pre-pulse. A further improvement of "proto \#2" $\mathrm{CO}_{2}$ laser power (from $8 \mathrm{~kW}$ to 12 $\mathrm{kW}$ at plasma point) is expected to follow the installation of the new pre-amplifier. Next target is a week-level source operation delivering $100 \mathrm{~W}$ clean EUV power with $\mathrm{CE}=3.5 \%$ and $12 \mathrm{~kW} \mathrm{CO}$ laser power.

$\mathrm{New}>20 \mathrm{~kW} \mathrm{CO}$ laser power amplifier system is under construction in co-operation with Mitsubishi Electric Corporation. The milestone of this development branch is shown in Table 2 as a "customer beta" system. We are planning to achieve EUV power generation with $\mathrm{CE}=4.5 \%$ using $20 \mathrm{~kW} \mathrm{CO} 2$ laser power by the end of Q2 of 2015 . Final target is a week-level operation producing a clean $250 \mathrm{~W}$ EUV power at the intermediate focus. Target shipment date of our first "customer beta" LPP light source unit is projected for 2015 .

\section{Acknowledgement}

This work was partly supported by the New Energy and Industrial Technology Development Organization (NEDO), Japan. We acknowledge their continuous support.

We acknowledge contributions of the following researchers and organizations. Plasma simulations were provided by Dr. Jun Sunahara, Dr. Katsunori Nishihara, Prof. Hiroaki Nishimura, and others in Osaka University. Plasma diagnostics was carried out by Dr. Kentaro Tomita, Prof. Kiichiro Uchino and others in Kyushu University. Laser engineering was supported by Dr. Akira Endo of HiLase Project (Prague) and Prof. Masakazu Washio and others in Waseda University. We also acknowledge many companies and engineers; EUV collector mirror collaboration by collector mirror suppliers - especially Rigaku for providing us with useful data. New $\mathrm{CO}_{2}$ laser amplifier development was done by Mitsubishi Electric $\mathrm{CO}_{2}$ laser team: Dr. Yoichi Tanino, Dr. Junichi Nishimae, Dr. Shuichi Fujikawa and others.

Authors were very sad to learn of a sudden and premature death of Dr. Yoichi Tanino on 1st February in 2014. We appreciate his extremely great job of $\mathrm{CO}_{2}$ amplifier development in a very short period of time and pray for his soul.

\section{References}

[1] Noreen Harned; Mieke Goethals; Rogier Groeneveld; Peter Kuerz; Martin Lowisch; Henk Meijer; Hans Meiling; Kurt Ronse; James Ryan; Michael Tittnich; Harm-Jan Voorma; John Zimmerman; Uwe Mickan; Sjoerd Lok.: 'EUV Lithography with the Alpha Demo Tools: status and challenges', Proc. SPIE 6517, (2007) 6517-06.

[2] Takaharu Miura, Katsuhiko Murakami, Kazuaki Suzuki; Yoshiaki Kohama, Kenji Morita, Kazunari Hada, Yukiharu Ohkubo, Hidemi Kawai,: 'Nikon EUVL development progress update', Proc. SPIE 6921, (2008) 6921-0M

[3] Christian Wagner, Noreen Harned, Peter Kuerz, Martin Lowisch, Hans Meiling, David Ockwell, Rudy Peeters, Koen van Ingen-Schenau, Eelco van Setten, Judon Stoeldraijer, Bernd Thuering: 'EUV into production with ASML's NXE platform', Proc. SPIE 7636, (2010) 7636-1H .

[4] Christian Wagner, Jose Bacelar, Noreen Harned, Erik Loopstra, Stef Hendriks, Ivo de Jong, (ASML Netherlands B.V., Netherlands), Peter Kuerz, (Carl Zeiss SMT AG, Germany); Leon Levasier, Mark van de Kerkhof, (ASML Netherlands B.V., Netherlands), Martin Lowisch, (Carl Zeiss SMT AG, Germany), Hans Meiling, David Ockwell, Rudy Peeters, Eelco van Setten, Judon Stoeldraijer, Stuart Young, John Zimmerman, Ron Kool, (ASML Netherlands B.V., Netherlands): 'EUV lithography at chipmakers has started: performance validation of ASML's NXE:3100', Proc. SPIE 7969, (2011) 7969-49.

[5] Hakaru Mizoguchi; Tamotsu Abe; Yukio Watanabe; Takanobu Ishihara; Takeshi Ohta; Tsukasa Hori; Akihiko Kurosu; Hiroshi Komori; Kouji Kakizaki; Akira Sumitani; Osamu Wakabayashi; Hiroaki Nakarai; Junichi Fujimoto; Akira Endo: 'First generation laser-produced plasma source system for HVM EUV lithography', Proc. SPIE 7636, (2010) 763608

[6] Akira Endo, Hideo Hoshino, Takashi Suganuma, Masato Moriya, Tatsuya Ariga, Yoshifumi Ueno, Masaki Nakano, Takeshi Asayama, Tamotsu Abe, Hiroshi Komori, Georg Soumagne, Hakaru Mizoguchi, Akira Sumitani, Koichi Toyoda, (Extreme Ultraviolet Lithography System Development Association, Japan): 'Laser produced EUV light source development for HVM', Proc. SPIE 6517, (2007) 6517-23.

[7] Hakaru Mizoguchi, Tamotsu Abe, Yukio Watanabe, Takanobu Ishihara, Takeshi Ohta, Tsukasa Hori, Tatsuya Yanagida, Hitoshi Nagano, Takayuki Yabu, Shinji Nagai, Georg Soumagne, Akihiko Kurosu, Krzysztof M. Nowak, Takashi Suganuma, Masato Moriya, Kouji Kakizaki, Akira Sumitani, Hidenobu Kameda, Hiroaki Nakarai, Junichi Fujimoto: '100W 1st Generation Laser-Produced Plasma light source system for HVM EUV lithography', Proc. SPIE 7969, (2011) 7969-08

[8] Katsunobu Nishihara, Akira Sasaki, Atsushi Sunahara, Takeshi Nishikawa: "EUV Sources for Lithography", Chap. 11, ed. V. Bakshi, SPIE Bellingham, (2005) 339-370.

[9] Hiroki Tanaka, Atsushi Matsumoto, Kouzi Akinaga, Akihiko Takahashi and Tatsuo Okada: "Comparative study on emission characteristics of extreme ultraviolet radiation from $\mathrm{CO}_{2}$ and $\mathrm{Nd}$ :YAG laser-produced tin plasmas', Appl. Phys. Lett. 87, (2005) 041503.

[10] Akira Endo, Hideo Hoshino, Takashi Suganuma, Krzysztof Nowak, Tatsuya Yanagida, Takayuki Yabu, Takeshi Asayama, Yoshifumi Ueno, Masato Moriya, Masaki Nakano, Hiroshi Someya, Toshihiro Nishisaka, Tamotsu Abe, Georg Soumagne, Hiroshi Komori, Hakaru Mizoguchi, Akira Sumitani, Koichi Toyoda: 'CO2 laser-produced Sn-plasma source for high-volume manufacturing EUV lithography', Proc. SPIE 6921, (2008) 6921-31.

[11] Tatsuya Yanagida, Hitoshi Nagano, Yasunori Wada, Taka- 
yuki Yabu, Shinji Nagai, Georg Soumagne, Tsukasa Hori, Kouji Kakizaki, Akira Sumitani, Junichi Fujimoto, Hakaru Mizoguchi, Akira Endo: 'Characterization and optimization of tin particle mitigation and EUV conversion efficiency in a laser produced plasma EUV light source', Proc. SPIE 7969, (2011) 7969-100.

[12] Junichi Fujimoto, Takeshi Ohta, Krzysztof M. Nowak, Takashi Suganuma, Hidenobu Kameda, Masato Moriya, Toshio Yokoduka, Koji Fujitaka, Akira Sumitani, Hakaru Mizoguchi: 'Development of the reliable $20 \mathrm{~kW}$ class pulsed carbon dioxide laser system for LPP EUV light source', Proc. SPIE 7969, (2011) 7969-99.

[13] H. Mizoguchi: 'High CE technology for HVM EUV source', Proc. of International Symposium on Extreme U1traviolet Lithography 2012, (2012) 353.

[14] Y. Tanino: 'A proposal for an EUV light source using transverse flow $\mathrm{CO}_{2}$ lasers', Proc. of International Symposium on Extreme Ultraviolet Lithography 2012, (2012) 1016.

[15] H. Mizoguchi: 'High CE and Magnetic Mitigation Technology for HVM EUV Source', Proc. of International Symposium on Extreme Ultraviolet Lithography 2013, (2013) S01 .

[16] Y. Tanino, J. Nishimae, T. Yamamoto, K. Funaoka, T. Tamida, S. Tsuda, S. Fujikawa, (Mitsubishi Electric Corporation, Japan), T. Ohta, Y. Kawasuji, (Gigaphoton Inc., Japan): 'A Driver $\mathrm{CO}_{2}$ Laser Using Transverse-flow $\mathrm{CO}_{2}$ Laser Amplifiers', Proc. of International Symposium on Extreme Ultraviolet Lithography 2013, (2013) S-2.

[17] Michael Kriese, Yuriy Platonov, Licai Jiang, Jim Rodriguez, (Rigaku Innovative Techologies), Ulrich Mueller, Jay Daniel, Shayna Khatri, Adam Magruder, (L-3 Communications), Steven Grantham, Charles Tarrio, Thomas Lucatorto, (National Institute of Standards and Technology): 'Develop- ment of EUVL Collector Technologies for Infrared Radiation Suppression', Proc. of International Symposium on Extreme Ultraviolet Lithography 2013, (2013) SC-01.

[18] K. M. Nowak, T. Ohta, T. Suganuma, J. Fujimoto, H. Mizoguchi, A. Sumitani, A. Endo: ' $\mathrm{CO}_{2}$ laser drives extreme ultraviolet nano-lithography - second life of mature laser technology'. Opto-Electron. Rev., 21(4), (2013) 52.

[19] Krzysztof M. Nowak, Takeshi Ohta, Takashi Suganuma, Junichi Fujimoto, and Hakaru Mizoguchi: 'Multi-line shortpulse solid-state seeded carbon-dioxide laser for extreme ultraviolet employing multi-pass radio-frequency excited slab amplifier'. Opt. Lett., 38(6), (2013), 881.

[20] Krzysztof M. Nowak: 'Solid-state seeded nano-second pulse $\mathrm{CO}_{2}$ oscillator for extreme ultraviolet lithography'. Photon. Lett. Poland, 5(3, ) (2013) 97.

[21] Krzysztof M. Nowak, Takeshi Ohta, Takashi Suganuma, Toshio Yokotsuka, Junichi Fujimoto, Hakaru Mizoguchi, and Akira Endo: 'Spectral characteristics of quantumcascade laser operating at $10.6 \mathrm{~mm}$ wavelength for a seed application in laser-produced-plasma extreme UV source'. Opt. Lett., 37(22), (2012) 4765.

[22] Krzysztof M. Nowak, Takeshi Ohta, Takashi Suganuma, Junichi Fujimoto, Hakaru Mizoguchi, Akira Sumitani, and Akira Endo: 'Efficient multiline nanosecond pulse amplification in planar waveguide $\mathrm{CO}_{2}$ amplifier for extreme UV laser-produced plasma source', Opt. Lett. 39(7), (2014) 1953.

[23] Yoichi Tanino, Junichi Nishimae, Tatsuya Yamamoto, Koji Funaoka, Taichiro Tamida, Shingo Tsuda, and Shuichi Fujikawa :'Transverse-flow radio-frequency-excited amplifier seeded by a cavity-dumped $\mathrm{CO}_{2}$ laser for an extreme ultraviolet light source', Opt. Lett. 38(17), (2013) 3291.

(Received: April 4, 2014, Accepted: May 2, 2016) 